\title{
Competition and fixation of cohorts of adaptive mutations under Fisher geometrical model
}

Jorge A. Moura de Sousa, João Alpedrinha, Paulo R. A. Campos, Isabel Gordo

One of the simplest models of adaptation to a new environment is Fisher's Geometric Model (FGM), in which populations move on a multidimensional landscape defined by the traits under selection. The predictions of this model have been found to be consistent with current observations of patterns of fitness increase in experimentally evolved populations. Recent studies investigated the dynamics of allele frequency change along adaptation of microbes to simple laboratory conditions and unveiled a dramatic pattern of competition between cohorts of mutations, i.e. multiple mutations simultaneously segregating and ultimately reaching fixation. Here, using simulations, we study the dynamics of phenotypic and genetic change as asexual populations under clonal interference climb a Fisherian landscape, and ask about the conditions under which FGM can display the simultaneous increase and fixation of multiple mutations - mutation cohorts - along the adaptive walk. We find that FGM under clonal interference, and with varying levels of pleiotropy, can reproduce the experimentally observed competition between different cohorts of mutations, some of which have a high probability of fixation along the adaptive walk. Overall, our results show that the surprising dynamics of mutation cohorts recently observed during experimental adaptation of microbial populations can be expected under one of the oldest and simplest theoretical models of adaptation - FGM. 


\section{Competition and fixation of cohorts of adaptive mutations under Fisher geometrical model}

2

3 Jorge A. Moura de Sousa ${ }^{1}$, João Alpedrinha ${ }^{1}$, Paulo R. A. Campos², Isabel Gordo ${ }^{1}$

4

$5{ }^{1}$ Instituto Gulbenkian de Ciência. Rua da Quinta Grande, 6. 2780-156 Oeiras, Portugal.

$6{ }^{2}$ Departamento de Física, Universidade Federal de Pernambuco, Cidade Universitaria, Recife

7 PE, Brazil.

8

$9 *$ Corresponding author

10 jasousa@igc.gulbenkian.pt

11 Telf: +351214407915

12 Fax: +351214407970

13

14

15

16

17

18

19

20

21

22

23 


\section{Abstract}

25 One of the simplest models of adaptation to a new environment is Fisher's Geometric Model 26 (FGM), in which populations move on a multidimensional landscape defined by the traits under 27 selection. The predictions of this model have been found to be consistent with current 28 observations of patterns of fitness increase in experimentally evolved populations. Recent studies 29 investigated the dynamics of allele frequency change along adaptation of microbes to simple 30 laboratory conditions and unveiled a dramatic pattern of competition between cohorts of 31 mutations, i.e. multiple mutations simultaneously segregating and ultimately reaching fixation. 32 Here, using simulations, we study the dynamics of phenotypic and genetic change as asexual populations under clonal interference climb a Fisherian landscape, and ask about the conditions under which FGM can display the simultaneous increase and fixation of multiple mutations mutation cohorts - along the adaptive walk. We find that FGM under clonal interference, and with varying levels of pleiotropy, can reproduce the experimentally observed competition between different cohorts of mutations, some of which have a high probability of fixation along the adaptive walk. Overall, our results show that the surprising dynamics of mutation cohorts recently observed during experimental adaptation of microbial populations can be expected under one of the oldest and simplest theoretical models of adaptation - FGM. 


\section{Introduction}

48 Understanding the mechanisms and dynamics underneath the adaptive process is still a great 49 challenge in evolutionary biology. Even in relatively simple environments, evolution 50 experiments demonstrate that this process often involves complex dynamics such as: (1) 51 competition between clones carrying different adaptive alleles (Desai \& Fisher, 2007; Perfeito et 52 al., 2007; Maharjan \& Ferenci, 2015), (2) hitchhiking, along with beneficial alleles, of neutral 53 and even deleterious mutations (Gerrish \& Lenski, 1998; Desai \& Fisher, 2007; Perfeito et al., 54 2007; Lang et al., 2013; Maharjan \& Ferenci, 2015), (3) second-order selection of mutations 55 which lead to increased mutation rates and mutator phenotypes (Sniegowski, Gerrish \& Lenski, 1997; Tenaillon et al., 2001; Desai, Fisher \& Murray, 2007; Perfeito et al., 2007; Barrick et al.,

57 2009; Wielgoss et al., 2013; Maharjan \& Ferenci, 2015), or (4) the emergence of negative 58 frequency-dependent interactions between genotypes (Gerrish \& Lenski, 1998; Maharjan, 2006; 59 Desai, Fisher \& Murray, 2007; Desai \& Fisher, 2007; Perfeito et al., 2007; Herron \& Doebeli, 60 2013; Lang et al., 2013; Maharjan \& Ferenci, 2015). It is increasingly evident that not only these dynamics influence the adaptive process but also that they emerge as a result of the adaptive

62 process. For instance, the fixation of mutator phenotypes has been typically observed in adapting populations, as their higher mutation rate provides them with a higher probability of acquiring and hitchhiking with rare beneficial mutations (Chao \& Cox, 1983; Taddei et al., 1997; Tanaka,

65 Bergstrom \& Levin, 2003; Desai \& Fisher, 2007; Gentile et al., 2011; Torres-Barceló et al., 2013; Lang et al., 2013). More recently, experimental findings from microbial evolution experiments coupled with sequencing analysis unveiled that a dramatic level of polymorphism in populations can occur during adaptation (Lang et al., 2013; Frenkel, Good \& Desai, 2014; 
70 mutations that segregate and reach fixation simultaneously, are observed in populations adapting

71 to simple environmental laboratory conditions. In large populations, the input of new mutations

72 can be so high that new mutants emerge in backgrounds already carrying other mutations,

73 leading to the formation and competition between mutation cohorts. Such competition results in

74 longer times for mutations to reach fixation and complex dynamics, as different mutations

75 aggregate in separate groups. Indeed, synchronous increase or decrease in frequency of these

76 mutations, competition between distinct cohorts and the simultaneous fixation of the mutations

77 that form the cohorts is a pervasive observation during this laboratory microbial adaptations

78 (Sniegowski, Gerrish \& Lenski, 1997; Tenaillon et al., 2001; Barrick et al., 2009; Wielgoss et al.,

79 2013; Lee \& Marx, 2013; Lang et al., 2013; Maddamsetti, Lenski \& Barrick, 2015).

80

81 A classical model of adaptation to a novel environment, theorized almost 100 years ago by

82 Fisher before the structure of DNA was discovered, is Fisher's Geometrical Model (FGM). It is a

83 simple model, where a population adapts towards a fixed phenotypic optimum (Fisher, 1930).

84 FGM considers the process of adaptation assuming that individuals are defined by their traits

85 under selection, which are geometrically represented in a defined multidimensional landscape. In

86 this model, directionality in selection emerges by assuming that fitness is related to the distance

87 of each phenotype to the optimum. Thus, a population moves towards the fitness peak through

88 the gradual accumulation of beneficial mutations. FGM has been extensively studied beyond its

89 original scope to make predictions under different scenarios about the distribution of beneficial

90 mutations during adaptation (Orr, 1998; Martin \& Lenormand, 2008; Bataillon, Zhang \& Kassen,

91 2011), the level of epistasis between mutations (Martin, Elena \& Lenormand, 2007; Blanquart et

92 al., 2014), the effects of deleterious mutations accumulated under relaxed selection (Martin \& 
93 Lenormand, 2006; Perfeito et al., 2014), the effect of drift load in the fitness at equilibrium (Otto

94 \& Orive, 1995; Lourenço, Galtier \& Glémin, 2011), sympatric speciation in an environment with

95 multiple fitness peaks (Barton, 2001; Sellis et al., 2011) and the effect of mutation pleiotropy

96 (the number of traits affected by a single mutation) in adaptation (Welch \& Waxman, 2003;

97 Chevin, Martin \& Lenormand, 2010; Lourenço, Galtier \& Glémin, 2011). Martin (Martin, 2014)

98 recently proposed that FGM basic assumptions can emerge from models which consider the

99 nature of complex metabolic networks within a cell. FGM predictions are largely compatible

100 with observations coming from experimental evolution studies, mostly in microorganisms

101 (MacLean, Perron \& Gardner, 2010; Chou et al., 2011; Khan et al., 2011; Sousa, Magalhaes \&

102 Gordo, 2012; Gordo \& Campos, 2013; Weinreich \& Knies, 2013; Tenaillon, 2014).

103

104 Here we ask whether the patterns of competition and fixation of simultaneous segregating 105 mutations (mutation cohorts) along an adaptive walk observed experimentally can be reproduced 106 under FGM. We study FGM under clonal interference by simulating populations with a large 107 mutational input ( $N U>>1$, where $N$ is the population size and $U$ the genomic mutation rate), 108 where both beneficial and deleterious mutations occur, therefore generating competing 109 polymorphisms (Gordo \& Campos, 2013). Since the simplest version of FGM assumes full 110 mutational pleiotropy, which is a restrictive assumption and thought to bear poor biological 111 realism (Welch \& Waxman, 2003; Orr, 2005; Chevin, Lande \& Mace, 2010; Wang, Liao \& 112 Zhang, 2010; Wagner \& Zhang, 2011; Lourenço, Galtier \& Glémin, 2011), we also studied a 113 model assuming partial pleiotropy. The degree of mutational pleiotropy is expected to influence 114 the dynamics of adaptation (Wagner \& Zhang, 2011). In our model of partial pleiotropy, similar 115 to that of (Lourenço, Galtier \& Glémin, 2011), a single mutation can only change a subset of 
116 traits $(m)$, taken at random from the full set of traits $(n)$ that contribute to fitness. When

117 populations have small sizes or mutation rates are low, the analytical expressions for predicting

118 the rate of adaptation under this model suggest that mutational pleiotropy can affect the

119 dynamics of adaptation of populations approaching a fitness peak (Lourenço, Galtier \& Glémin,

120 2011). However, such analytical results rely on a strong simplifying assumption: the populations

121 are monomorphic most of the time. This assumption is quite restrictive given the increasing

122 experimental evidence for high rates of beneficial mutations both in natural (Eyre-Walker \&

123 Keightley, 2007; Jensen, Thornton \& Andolfatto, 2008) and in experimental populations

124 (Perfeito et al., 2007; Good, Rouzine \& Balick, 2012), which promptly produce competition

125 between segregating mutations arising in distinct lineages and drive the dynamics of mutation

126 cohorts described above. To address these more relevant scenarios, we use stochastic simulations

127 of FGM for populations undergoing strong clonal interference. We consider large populations

128 and values of mutation rate and mean effect of mutations that are in reasonable agreement with

129 current estimates for microbial populations (Gordo, Perfeito \& Sousa, 2011; Perfeito et al., 130 2014).

131 Most of theoretical analysis done so far focused on predicting the equilibrium mean fitness, and

132 did not address the time scale at which such equilibrium is in fact reached. As experiments where

133 evolution is followed for longer and longer periods are emerging (Lang et al., 2013; Barrick \&

134 Lenski, 2013), it is also important to have theoretical expectations on the full dynamics of the

135 approach to equilibrium under classical models of adaptation, both at the phenotypic and 136 genotypic level, as we do here. By tracking each individual mutation during the adaptive walk as

137 the populations approach the optimum, we find that the simplest version of FGM can generate 
138 the complex mutation cohort dynamics observed in microbial adaptation experiments, under

139 specific evolutionary parameters within a biological realistic range.

140

\section{Methods}

\section{Simulation Methods of Fisher Geometrical Model}

143 FGM considers each individual as a point in a $n$-dimensional space, where $n$ is the number of

144 traits under selection. Each individual is characterized by a vector of coordinates $\left(z_{1}, z_{2}, \ldots, z_{\mathrm{n}}\right)$

145 that gives the position of the individual in the fitness landscape. This vector represents the

146 phenotypic values for each trait. Without loss of generality, we define the optimum as the origin

147 of the $n$-dimensional space. As commonly done, we assume that fitness is given by a Gaussian

148 function of the distance to the optimum, $w=\exp \left(-\sum_{i=1}^{n} z_{i}^{2}\right)$. We assume that mutations, as rare

149 events, follow a Poisson distribution with a genomic mutation rate $U$, per individual, per

150 generation. Each mutation changes $m$ traits chosen at random from the total $n$ traits, and the

151 effect it causes in each affected trait follows a normal distribution with mean 0 and variance $\sigma^{2}$.

152 We consider a Wright-Fisher model and assume multinomial sampling with fixed population size

$153 N$. The contribution of each individual to the next generation is proportional to its fitness and it is

154 based on a multinomial sampling of the population. We assume large population sizes, as typical

155 in microbial laboratory adaptation experiments (Barrick \& Lenski, 2013), and consider values of

156 genomic mutation rates $(U)$ that are reasonable for microbes. Indeed the order of magnitude of

157 the genomic mutation rate has been previously estimated for many species of organisms and falls

158 within $U \sim 0.001$ (Drake et al., 1998; Lee, Popodi \& Tang, 2012). We explored values for the

159 complexity within a range that is in accordance with estimates obtained from experiments using

160 different organisms including viruses, bacteria and multicellular organisms (reviewed in 
161 (Lourenço, Galtier \& Glémin, 2011), specifically Figure 2 of that paper). We also explored

162 different values of the mean effect of mutations $\left(\mathrm{E}(\mathrm{S})=-m \sigma^{2}\right.$, under the assumptions of partial

163 pleiotropy in FGM, described above) ranging from very small $-0.1 \%$, to much larger $(-20 \%)$, as

164 estimated from different mutation accumulation experiments in different organisms (Martin \&

165 Lenormand, 2006; Eyre-Walker \& Keightley, 2007; Gordo, Perfeito \& Sousa, 2011). The code

166 for the simulations is provided as supplementary material (Supplemental Files 1, 2, 3 and 4).

167

168 Results

169 Dynamics of approach to equilibrium mean fitness

170 We start by studying the dynamics of fitness increase along tens of thousands of generations for

171 different levels of phenotypic complexity $(n)$, pleiotropy $(m)$ and mean effect mutations $(\mathrm{E}(\mathrm{S}))$.

172 Figure 1A shows that the initial rate of fitness increase is lower under low pleiotropy across all

173 values of the mean fitness effect of mutations $(\mathrm{E}(\mathrm{S}))$ studied. The effect is particularly strong for

$174|\mathrm{E}(\mathrm{S})|>0.01$. However, in the long run populations with lower pleiotropy reach higher levels of

175 mean fitness (see also Supplementary Figure S1). Increasing complexity, while maintaining a

176 similar level of pleiotropy, shows a similar pattern for the fitness plateau, where we find that

177 populations with fewer traits reach higher fitness values within the simulated time period

178 (Figure 1B).

179 Mutation cohorts of fixed in the initial steps of adaptation

180 Next, we studied the dynamics of mutation fixation along the adaptive walk. We first studied 181 populations with maximum pleiotropy and various degrees of complexity across different E(S)

182 and mutation rates, and asked how many mutations fix simultaneously in the first step, i.e. the

183 mutation cohort size at the first fixation step. Figure 2 shows that fixations of cohorts of 
184 mutations can be very common, reflecting the degree of clonal interference occurring in these

185 large populations. Across all parameters, the major determinant of the number of mutations

186 fixing in cohorts is the mean effect of mutations $(\mathrm{E}(\mathrm{S}))$, with lower effect mutations promoting

187 fixation of cohorts of larger size. The other relevant parameter to the size of the fixed cohorts is,

188 as expected, the mutation rate, with an increased mutation rate showing the largest cohorts of

189 mutations fixed. Therefore, the combination of small effect mutations generated at a high rate

190 leads to the fixation of larger mutation cohorts. We performed the same analysis on simulations

191 where we relax the assumption of full pleiotropy. Populations with partial pleiotropy $(m=3,10$ or

192 20) for the highest level of complexity previously tested $(n=30)$ show patterns that are

193 qualitatively similar (Supplementary Figure S2). The main difference detected occurs in

194 simulations with a high mutation rate, where the likelihood of observing large stronger effect

195 mutation cohorts increases relative to the case of full pleiotropy. Additionally, both in the cases

196 of full or partial pleiotropy, the complexity of evolving populations shows a minimal effect on

197 the size of the fixed mutation cohorts. Therefore, the number of mutations observed fixing

198 simultaneously in the first step of adaptation is mainly determined by the mutation rate and the

199 mean selective effect of mutations.

200

201

202 Mutation cohorts fixed along the adaptive walk

203 In order to understand how the probability of observing the fixation of mutation cohorts changes

204 along the adaptive walk, we next study the distribution of mutations fixed beyond the first step of

205 adaptation. Figure 3 shows the pattern of mutation cohorts fixed along an adaptive walk lasting

20630000 generations. Each point in the panels of Figure 3 corresponds to a fixation event 
207 occurring during this time period, with the number of mutations (i.e., the size of the cohorts) that

208 compose each of these fixations represented in the y-axis. The probability of observing cohorts

209 consisting of a large number of mutations later in the adaptive walk is strongly dependent on the

210 average selective effect of the mutations (contrast left panels with right panels in Figure 3).

211 Lower effect mutations lead to the fixation of cohorts of larger sizes not only in the first steps,

212 but also as populations approach the equilibrium fitness. Interestingly, we observe that, for the

213 lower values of mean mutation effect, the likelihood of fixing mutation cohorts of larger sizes

214 (from 4 to 8 mutations) increases for populations with a higher complexity, throughout the

215 adaptive walk. For high values of $|\mathrm{E}(\mathrm{S})|$ and populations with a lower number of traits, fixation

216 of large mutation cohorts becomes an increasingly rare event once they approach the fitness

217 equilibrium.

218 Overall, along the adaptive walk, the size of fixed mutation cohorts tends to shrink, at a faster

219 pace for large values of $|\mathrm{E}(\mathrm{S})|$. These simulations therefore suggest that, for long-term adaptation

220 of populations approaching a fixed optimum, fixation of single mutations is expected to become

221 the dominant pattern. However, when $|\mathrm{E}(\mathrm{S})|$ is small (left panels in Figure 3, where $|\mathrm{E}(\mathrm{S})|$ is

2220.001 or 0.003 ) that regime may take a substantial time to be reached.

223 We have also explored the role of partial pleiotropy on the size of mutation cohorts fixed along

224 the adaptive walk. The results are qualitatively similar to the ones observed for populations with

225 full pleiotropy, with the size of mutation cohorts generally decreasing as populations get closer

226 to the fitness peak, but the decrease taking longer periods of time as $|\mathrm{E}(\mathrm{S})|$ becomes smaller (see

227 Supplementary Figure S3A). Furthermore, the distributions of cohort sizes of the mutations

228 simultaneously fixed along the adaptive walks, for populations under partial and full pleiotropy, 
229 are similar, as shown in Supplementary Figure S3B. Overall, these observations indicate that

230 partial pleiotropy plays a minor contribution to the fixation of multiple mutations.

232 Dynamics of mutation cohorts

233 Finally, we study the dynamics of polymorphism expected in populations climbing the Fisherian

234 landscape. We first focus our simulations on short-term evolution, a time scale for which 235 polymorphism data has been obtained recently for yeast strains adapting to a simple laboratory 236 environment (Lang et al., 2013). Figure 4 shows the dynamics of frequency change of each 237 individual mutation segregating in populations evolving for 1000 generations. Aggregation of 238 cohorts of mutations can be clearly observed across the different replicate populations, all 239 simulated with the same evolutionary parameters. The parameter set shown was chosen to be one 240 where we could find a pattern similar to that observed in the evolution experiments done in yeast

241 (Lang et al., 2013). In the replicate simulated populations, just as in the replicate experimental 242 ones, cohorts of different sizes emerge and compete against each other, with some achieving 243 fixation and others being outcompeted. Although this phenomenon of "cohort interference" is 244 more likely for cohorts competing at lower frequencies (where many mutations are segregating), 245 it can also be observed when mutations reach high frequencies (e.g., first and third panels in the 246 first row). Even under the same parameter values different patterns can be observed among the 247 replicates: sequential fixation of cohorts of low size in some populations (e.g., fourth and 248 seventh panels) and fixation of cohorts of large size in other populations (e.g., fifth, sixth and 249 ninth panels). The same qualitative behavior is observed when simulating a higher number of 250 replicate populations adapting under FGM (see Supplementary Figure S4). 
251 The formation of mutation cohorts under FGM can be observed across several parameter sets,

252 under different values of complexity and mean mutation effects (see Supplementary Figure S5

253 for examples). Yet some sets of parameters lead to results more consistent with the observations

254 originating from evolution experiments than others. In the yeast evolving populations analyzed

255 in (Lang et al., 2013) an average of 25 different mutations segregating and an average of 6

256 fixations were observed, across the replicate populations. Moreover, considerable fitness

257 increases could be detected (around $\sim 6 \%$ after 500 generations). Across the sets of parameters

258 we explored, this level of fitness increase and high number of mutations was unlikely when

259 simulating FGM with high complexity $(n=90$, rightmost panels in Supplementary Figure S5A

260 and B). Moreover, out of 40 independently evolving populations in (Lang et al., 2013), in all but

261 one replicate more than two mutation cohorts were observed to be segregating (population

262 BYB1-B01). A pattern with few mutation cohorts was detected in the simulations sets we

263 performed under full pleiotropy, low complexity and strong effect mutations (leftmost top panel

\section{4 in Supplementary Figure S5A).}

265 Under the parameter values assumed in Figure 4, we followed the dynamics of frequency 266 change beyond 1000 generations to ask about the extent to which polymorphisms would be lost 267 in the long run. Remarkably the simulations indicate that polymorphisms can be maintained for a 268 very long time (sometimes more than 10000 generations) (see Supplementary Figure S6). The 269 simulations also indicate that the emergence of new mutation cohorts of large size becomes less 270 frequent than that observed during the first 1000 generations. Data from experiments involving 271 longer time periods may thus help determining if the predictions of this model are met.

272

\section{Discussion}


274 With the advances of next generation sequencing, increased power to observe the dynamics of

275 adaptation at the resolution of individual mutations has emerged. The data recently gathered

276 indicates that adaptation of microbial populations adapting in laboratory environments exhibit

277 patterns very distinct from the classic single selective sweep model of periodic selection. Instead

278 the dynamics of molecular evolution in these microbes evolving in real time shows that

279 aggregates of beneficial mutations segregate and fix simultaneously (Lang et al., 2013;

280 Maddamsetti, Lenski \& Barrick, 2015; Zanini et al., 2016). Even though Fisher's model is a

281 phenotypic model of adaptation, the easiness by which this simple non-gene centric model is

282 able to produce dynamics of fitness change similar to those observed in such experiments, lead

283 us to ask if such dynamics of molecular change could be expected under this model. The

284 simulations performed show that Fisher's Geometric Model, in its simplest version, can

285 reproduce dynamics of cohort interference such as the ones observed in experimental settings. As

286 observed for the frequencies of sequenced mutations in evolve and re-sequence experiments in

287 yeast and bacteria, the mutation dynamics of simulated populations under FGM can be non-

288 monotonic and exhibit patters of interference between clones belonging to distinct mutation

289 cohorts. The number of mutations that compose these cohorts are found to be variable and the

290 polymorphisms emerging can last for thousands of generations. We note that FGM does not

291 consider social and ecological interactions that are likely to be important in explaining genetic

292 diversity in natural populations (Cordero \& Polz, 2014), nor does it consider frequency

293 dependent selection, which has been shown to also occur in laboratory evolving microbial

294 populations adapting to simple ecological conditions (Maharjan et al., 2012; Herron \& Doebeli, 295 2013). 
296 Remarkably, when simulating the dynamics of individual mutations produced under FGM, we

297 could find parameter sets leading to patterns very similar to the ones that are increasingly being 298 assayed through whole genome sequencing of evolving microbial populations (e.g., compare 299 Figure 4 with Figure 1 in (Lang et al., 2013)). Although such patterns are dependent on the 300 parameters used (see Supplementary Figure S5), they could be observed in simulated 301 populations assuming a set of parameters within a biological plausible range: a mean effect of 302 mutations around 1\%, consistent with measurements in microbes (Kibota \& Lynch, 1996; Zeyl $303 \&$ DeVisser, 2001), and a genomic mutation rate of $3 \times 10^{-3}$, consistent with Drake's rule (Drake 304 et al., 1998; Lee, Popodi \& Tang, 2012).

305 Under FGM, cohort interference can be common during the initial steps of adaptation, and is 306 more likely when the mean effect of mutations is small and mutation rates are not too small. In 307 these scenarios many small effect mutations simultaneously segregate, each taking a long time to 308 reach fixation, which likely results in the acquisition of additional mutations (either beneficial, 309 neutral or slightly deleterious) in the same genetic background. In contrast, when $\mathrm{E}(\mathrm{S})$ is large, 310 beneficial mutations sweep to fixation faster, and the likelihood of acquiring additional 311 mutations in their background diminishes. As expected the size of the interfering cohorts 312 increases as the mutation rate increases, since an increased amount of mutations segregating in 313 these high $U$ populations prevents the fast fixation of a single mutation.

314 Given that levels of complexity and pleiotropy may differ across genomes and environments, we 315 further enquired if the patterns described above would change for populations where differences 316 between complexity and pleiotropy are very large. We thus performed simulations with $n=500$ 317 and a low degree of pleiotropy $(m=3)$ for different values of $\mathrm{E}(\mathrm{S})$. Adaptation in these scenarios 318 occurs substantially slower, due to the very high dimensionality of the fitness landscape 
319 (Supplementary Figure S7A). The sizes of mutation cohorts initially fixed can also be large

320 (when $|\mathrm{E}(\mathrm{S})|$ is small), similarly to the simulations under lower complexity (Supplementary

321 Figure S7B). However, fixations now involve long waiting times, often more than 2000

322 generations (Supplementary Figure S7C). The simulations also indicate that fitness increase

323 resulting from the fixation of mutation cohorts can be very small (Supplementary Figure S7D).

324 Thus, both data of mutation frequency dynamics and of fitness increase along time are required

325 to determine the levels of complexity of the fitness landscape.

326 The relationship between the size of cohorts and both the mean effect of mutations and the

327 mutation rate is also detected when we study adaptation over longer periods (Figure 3). The size

328 of fixed cohorts tends to shrink along the adaptive walk, and does so at a faster pace for large

329 values of $\mathrm{E}(\mathrm{S})$. Therefore, for populations approaching a fixed optimum the pattern of long-term

330 adaptation is expected to become dominated by fixation of single mutations. However if $\mathrm{E}(\mathrm{S})$ is

331 small such pattern may take many thousands of generations to be detected (right panel in Figure

332 3), a time scale that is out of reach for most laboratory experiments so far studied. The famous

333 long-term evolution experiment (LTEE) in Escherichia coli constitutes an important exception,

334 where patterns of adaptation can be studied over periods as long as 60000 generations (Lenski et

335 al., 1991; Maddamsetti, Lenski \& Barrick, 2015). The access to samples frozen every 500

336 generations allows the tracking of individual mutations and the reconstruction of the

337 evolutionary genetic history of an individual population. Maddamsetti et al tracked the

338 emergence of 42 mutations in one of the evolving populations and showed competition and

339 interference between lineages carrying several mutations, including the simultaneous fixation of

340 these sets (Maddamsetti, Lenski \& Barrick, 2015). In this population however, not only clonal

341 interference was observed but also frequency-dependent selection was important in driving the 
342 dynamics of mutation cohorts. On a shorter-term experiment also with $E$. coli but now evolving

343 in a chemostat Maharjan and colleagues detected synchronous sweep of multiple mutations but

344 the levels of polymorphism were also driven by frequency dependent interactions between clones

345 (Maharjan et al., 2015). As we show here clonal interference alone can lead to dynamics of

346 cohort interference, but given the emergence of frequency dependent selection even in the

347 simplest environments, as well as its potential critical role in natural microbial populations

348 (Cordero \& Polz, 2014), it will be important in future work to model other fitness landscapes

349 which can allow for the simultaneous occurrence of both processes.

350

\section{Conclusions}

352 In the current work, we study a simple version of the Fisher's Geometrical Model that assumes

353 partial or full pleiotropy. Despite its simplicity, FGM has been successfully used to reproduce

354 patterns of the dynamics of the adaptive process (Chevin, Martin \& Lenormand, 2010; Martin,

355 2014). A common pattern emerging from the short-term dynamics of populations of

356 microorganisms evolving in laboratory conditions is the finding that mutants carrying multiple

357 segregating mutations can go to fixation (Lang et al., 2013; Maddamsetti, Lenski \& Barrick,

358 2015). Before resorting to more complex models of fitness landscapes (Cordero \& Polz, 2014),

359 we inquired whether a simple and less parameterized model, such as FGM, could capture the

360 essence of this sort of observation under reasonable parameters. Assuming large population

361 sizes close to those in the experiments, and mutation rates typical of microbes, thus naturally

362 driving population to a clonal interference regime, we show that FGM, both under full and

363 partial pleiotropy, generates patterns of segregation and competition of cohorts of mutations that

364 are consistent with experimental observations. 


\section{Acknowledgements}

367 IG thanks Olivier Tenaillon for pointing out the potential importance of partially pleiotropy 368 leading to different patterns of mutation accumulation.

\section{Figure Legends}

372 Figure 1 Dynamics of mean fitness increase under FGM with partial pleiotropy

373 A) Dynamics of mean fitness of asexual populations with varying degrees of pleiotropy $(m)$. All 374 populations have high complexity $(n=96)$ and distributions of fitness effects (DFEs) with 375 different means are studied. Other parameters are population size $N=10^{4}$, mutation rate $U=0.001$, 376 initial fitness $w_{0}=0.5$. The variance for the mutation effects $\sigma^{2}$ varies as $m$ varies, so that $\mathrm{E}(\mathrm{S})$ 377 (which is $-m \sigma^{2}$ in this model) has the value indicated in each panel.

378 B) The effect of increasing phenotypic complexity (n) on the dynamics of fitness increase. Other 379 population parameters are: $N=10^{4}, U=0.001$, variance $\sigma^{2}=0.004, m=3$ and the initial fitness $380 w_{0}=0.5$. Short-term dynamics are highlighted as an inset.

Figure 2 FGM can lead to simultaneous fixation of mutation cohorts. The probability 383 distribution of the number of mutations fixed during the first fixation event in the adaptive walk, 384 i.e. mutation cohort size. Parameter values used in these simulations are as follows: population 385 size $N=10^{4}$ and initial fitness $w_{0}=0.5$ are the same across all panels; the other parameters vary as 386 indicated in each panel (mutation rate $U$ increases bottom to top from 0.0001 to 0.01 , implying 
387 higher levels of clonal interference; phenotypic complexity $n$ (and pleiotropy $m=n$ ) increase from

388 left to right; and, within each panel, $\sigma^{2}$ varies, implying different distributions of arising 389 mutations with mean selective effects of $E(S)$ indicated by the different shades). Data is shown 390 for 100 simulations per combination of parameters.

391

392

393

394

395

396

397

398

399

400

401

402

403 404 405 406

407 408 409 410

Figure 3 Number of mutations fixed (mutation cohort size) along the adaptive walk. Parameter values are population size $N=10^{4}$, the initial fitness $w_{0}=0.5$, mutation rate $U=0.001$ and other parameters as indicated in each panel (with phenotypic complexity $n$ and mean effect of mutations $E(S)$ taking the values indicated on the left and above panels, respectively). In all panels full pleiotropy is assumed $(m=n)$. Data is shown for 100 simulations per combination of parameters.

Figure 4 Dynamics of frequency change of individual mutations along time across independently evolving populations. Aggregation of multiple mutations in cohorts can be easily detected by the simultaneous increase in frequency of different mutations (in different colors). Competition between cohorts can be commonly observed during the first 1000 generations of adaptation. Parameter values used are population size $N=10^{5}$, initial fitness $w_{0}=0.5$, mutation rate $U=0.003, n=10, m=3$ and $E(S)=-0.012$. Gaussian noise (mean 0, variance 0.02), mimicking experimental error, was added to the dynamics for increasing visibility and comparison with experimental data.

Supplementary Figure S1 Long term dynamics of mean fitness of populations as a function of the mean effect of mutations $(\mathbf{E}(\mathbf{S}))$. Dynamics are shown for high and low levels of pleiotropy $(m=96$ and $m=3)$. Other parameters are as in Figure 1. 
412 Supplementary Figure S2 Effect of pleiotropy in the simultaneous fixation of cohorts of 413 mutations during the first step of adaptation. The complexity, $n$, is fixed at 30 while the 414 pleiotropy, $m$, varies between 3, 10 and 20. Remaining parameters are as in Figure 2.

Supplementary Figure S3 Simulations showing the effect of pleiotropy on the dynamics of fixation of mutations along long periods of time. A) The complexity, $n$, is fixed at 30 , while the pleiotropy, $m$, varies between 3,10 and 20. Remaining parameters are as in Figure 3. B)

419 Histograms with frequencies of the number of mutations fixed in each cohort, across the 30000 420 generations, for populations with full $(n=3, m=3$, blue distributions) or partial ( $n=30, m=3$, red 421 distributions) pleiotropy. Shown are the distributions for populations with different mean effect 422 of mutations $(E(S))$. For each $E(S)$, Chi-square $\left(\chi^{2}\right)$ test for comparison of the distributions under 423 partial or full pleiotropy is shown.

Supplementary Figure S4 Additional simulations showing the dynamics of frequency change of individual mutations along time. The diverse patterns of cohorts observed emerge from the dynamics of adaptation under FGM. Parameters are as in Figure 4.

Supplementary Figure S5 Effect of complexity, pleiotropy and mean effect of mutations in the dynamics of frequency change of individual mutations along time. A) Two representative

431 simulations for each combination of parameters (all other parameters are as in Figure 4): 432 complexity $(n)$ increases from left $(n=3)$ to right $(n=90)$, with a fixed $m=3$; mean effect of 433 mutation $E(S)$ decreases from top $(E(S)=-0.012)$ to bottom $(E(S)=-0.0012)$. B) Fitness increase 
434 for one of the two representative populations of each panel, for the same combination of

435 parameters as shown in A.

436

437 Supplementary Figure S6 Long-term dynamics of frequency change of individual

438 mutations. Three representative dynamics of the patterns of cohorts observed during 10000

439 generations, indicating that under FGM polymorphisms can be maintained in populations for

440 large periods of time. Parameters used are as in Figure 4.

441

442 Supplementary Figure S7 Adaptation and cohort fixation in high dimensionality

443 phenotypic landscapes. Simulations of adapting populations with high complexity $(n=500)$ and

444 low pleiotropy $(m=3)$. Other parameters are: population size $N=10^{4}$, initial fitness $w_{0}=0.5$ and

445 mutation rate $U=0.001$. A) Dynamics of fitness increase for populations with varying mean

446 effect of mutations. B) Probability distribution of the number of mutations fixed during the first

447 fixation event (i.e. mutation cohort size). C) Distribution of the number of generations until the

448 first fixation. D) Distribution of average fitness of populations at the first fixation event. In A, a

449 single, representative simulation was used to assess the dynamics. B, C and D are the summary

450 of 100 replicate simulations.

451

452 References

453

454

455

456

457

458

459

460
Barrick JE, Lenski RE 2013. Genome dynamics during experimental evolution. Nature Publishing Group 14:827-839. DOI: 10.1038/nrg3564.

Barrick JE, Yu DS, Yoon SH, Jeong H, Oh TK, Schneider D, Lenski RE, Kim JF 2009. Genome evolution and adaptation in a long-term experiment with Escherichia coli. Nature 461:12431247. DOI: $10.1038 /$ nature08480.

Barton NH 2001. The role of hybridization in evolution. Molecular Ecology.

Bataillon T, Zhang T, Kassen R 2011. Cost of Adaptation and Fitness Effects of Beneficial 
461

462

463

464

465

466

467

468

469

470

471

472

473

474

475

476

477

478

479

480

481

482

483

484

485

486

487

488

489

490

491

492

493

494

495

496

497

498

499

500

501

502

503

504

505

506

Mutations in Pseudomonas fluorescens. Genetics 189:939-949. DOI: 10.1534/genetics. 111.130468.

Blanquart F, Achaz G, Bataillon T, Tenaillon O 2014. Properties of selected mutations and genotypic landscapes under Fisher's geometric model. Evolution 68:3537-3554.

Chao L, Cox EC 1983. Competition between high and low mutating strains of Escherichia coli. Evolution 37:125-134.

Chevin L-M, Lande R, Mace GM 2010. Adaptation, Plasticity, and Extinction in a Changing Environment: Towards a Predictive Theory. PLoS Biology 8:e1000357. DOI: 10.1371/journal.pbio.1000357.s001.

Chevin L-M, Martin G, Lenormand T 2010. Fisher's model and the genomics of adaptation: restricted pleiotropy, heterogeneous mutation and parallel evolution. Evolution 64:32133231. DOI: 10.1111/j.1558-5646.2010.01058.x.

Chou HH, Chiu HC, Delaney NF, Segre D, Marx CJ 2011. Diminishing Returns Epistasis Among Beneficial Mutations Decelerates Adaptation. Science 332:1190-1192. DOI: 10.1126/science. 1203799.

Cordero OX, Polz MF 2014. Explaining microbial genomic diversity in light of evolutionary ecology. Nature Reviews Microbiology. DOI: 10.1038/nrmicro3218.

Desai MM, Fisher DS 2007. Beneficial Mutation Selection Balance and the Effect of Linkage on Positive Selection. Genetics 176:1759-1798. DOI: 10.1534/genetics.106.067678.

Desai MM, Fisher DS, Murray AW 2007. The Speed of Evolution and Maintenance of Variation in Asexual Populations. Current Biology 17:385-394. DOI: 10.1016/j.cub.2007.01.072.

Drake JW, Charlesworth B, Charlesworth D, Crow JF 1998. Rates of spontaneous mutation. Genetics 148:1667-1686.

Eyre-Walker A, Keightley PD 2007. The distribution of fitness effects of new mutations. Nature Publishing Group 8:610-618. DOI: 10.1038/nrg2146.

Fisher RA 1930. The Genetical Theory of Natural Selection. Clarendon Press, Oxford.

Frenkel EM, Good BH, Desai MM 2014. The fates of mutant lineages and the distribution of fitness effects of beneficial mutations in laboratory budding yeast populations. Genetics. DOI: 10.1534/genetics.113.160069/-/DC1.

Gentile CF, Yu S-C, Serrano SA, Gerrish PJ, Sniegowski PD 2011. Competition between highand higher-mutating strains of Escherichia coli. Biology Letters 7:422-424. DOI: 10.1098/rsbl.2010.1036.

Gerrish PJ, Lenski RE 1998. The fate of competing beneficial mutations in an asexual population. Genetica 102-103:127-144.

Good BH, Rouzine IM, Balick DJ 2012. Distribution of fixed beneficial mutations and the rate of adaptation in asexual populations. DOI: 10.1073/pnas.1119910109/-/DCSupplemental.

Gordo I, Campos PRA 2013. Evolution of clonal populations approaching a fitness peak. Biology Letters 9:20120239. DOI: 10.1098/rsbl.2012.0239.

Gordo I, Perfeito L, Sousa A 2011. Fitness Effects of Mutations in Bacteria. Journal of Molecular Microbiology and Biotechnology 21:20-35. DOI: 10.1159/000332747.

Herron MD, Doebeli M 2013. Parallel evolutionary dynamics of adaptive diversification in Escherichia coli. PLoS Biology 11:e1001490. DOI: 10.1371/journal.pbio.1001490.

Jensen JD, Thornton KR, Andolfatto P 2008. An Approximate Bayesian Estimator Suggests Strong, Recurrent Selective Sweeps in Drosophila. PLoS Genetics 4:e1000198. DOI: 10.1371/journal.pgen.1000198.s005.

Khan AI, Dinh DM, Schneider D, Lenski RE, Cooper TF 2011. Negative Epistasis Between 
507

508

509

510

511

512

513

514

515

516

517

518

519

520

521

522

523

524

525

526

527

528

529

530

531

532

533

534

535

536

537

538

539

540

541

542

543

544

545

546

547

548

549

550

551

552

Beneficial Mutations in an Evolving Bacterial Population. Science 332:1193-1196. DOI: 10.1126/science. 1203801.

Kibota TT, Lynch M 1996. Estimate of the genomic mutation rate deleterious to overall fitness in E. coli. Nature:694-696. DOI: 10.1038/381694a0.

Lang GI, Rice DP, Hickman MJ, Sodergren E, Weinstock GM, Botstein D, Desai MM 2013. Pervasive genetic hitchhiking and clonal interference in forty evolving yeast populations. Nature:1-6. DOI: 10.1038/nature12344.

Lee H, Popodi E, Tang H 2012. Rate and molecular spectrum of spontaneous mutations in the bacterium Escherichia coli as determined by whole-genome sequencing. Proceedings of the National Academy of Sciences of the United States of America 2774-2783. DOI: 10.1073/pnas. 1210309109.

Lee M-C, Marx CJ 2013. Synchronous waves of failed soft sweeps in the laboratory: remarkably rampant clonal interference of alleles at a single locus. Genetics 193:943-952. DOI: 10.1534/genetics.112.148502.

Lenski RE, Rose MR, Simpson SC, Tadler SC 1991. Long-term experimental evolution in Escherichia coli. I. Adaptation and divergence during 2,000 generations. American Naturalist 138:1315-1341.

Lourenço J, Galtier N, Glémin S 2011. Complexity, pleiotropy, and the fitness effect of mutations. Evolution 65:1559-1571. DOI: 10.1111/j.1558-5646.2011.01237.x.

MacLean RC, Perron GG, Gardner A 2010. Diminishing Returns From Beneficial Mutations and Pervasive Epistasis Shape the Fitness Landscape for Rifampicin Resistance in Pseudomonas aeruginosa. Genetics 186:1345-1354. DOI: 10.1534/genetics.110.123083.

Maddamsetti R, Lenski RE, Barrick JE 2015. Adaptation, Clonal Interference, and FrequencyDependent Interactions in a Long-Term Evolution Experiment with Escherichia coli. Genetics 200:619-631. DOI: 10.1534/genetics.115.176677.

Maharjan R 2006. Clonal Adaptive Radiation in a Constant Environment. Science 313:514-517. DOI: $10.1126 /$ science. 1129865.

Maharjan R, Ferenci T 2015. Mutational signatures indicative of environmental stress in bacteria. Molecular Biology and Evolution 32:380-391. DOI: 10.1093/molbev/msu306.

Maharjan RP, Ferenci T, Reeves PR, Li Y, Bin Liu, Wang L 2012. The multiplicity of divergence mechanisms in a single evolving population. Genome Biology 13:R41. DOI: 10.1186/gb-2012-13-6-r41.

Maharjan RP, Liu B, Feng L, Ferenci T, Wang L 2015. Simple phenotypic sweeps hide complex genetic changes in populations. Genome Biology and Evolution 7:531-544. DOI: $10.1093 / \mathrm{gbe} / \mathrm{evv} 004$.

Martin G 2014. Fisher's geometrical model emerges as a property of complex integrated phenotypic networks. Genetics 197:237-255. DOI: 10.1534/genetics.113.160325.

Martin G, Lenormand T 2006. A general multivariate extension of Fisher's geometrical model and the distribution of mutation fitness effects across species. Evolution 60:893-907.

Martin G, Lenormand T 2008. The Distribution of Beneficial and Fixed Mutation Fitness Effects Close to an Optimum. Genetics 179:907-916. DOI: 10.1534/genetics.108.087122.

Martin G, Elena SF, Lenormand T 2007. Distributions of epistasis in microbes fit predictions from a fitness landscape model. Nature Genetics 39:555-560. DOI: 10.1038/ng1998.

Orr HA 1998. The population genetics of adaptation: the distribution of factors fixed during adaptive evolution. Evolution:935-949.

Orr HA 2005. Theories of adaptation: what they do and don't say. Genetica 123:3-13. 
553 Otto SP, Orive ME 1995. Evolutionary consequences of mutation and selection within an 554 individual. Genetics 141:1173-1187.

555

556

557

558

559

560

561

562

563

564

565

566

567

568

569

570

571

572

573

574

575

576

577

578

579

580

581

582

583

584

585

586

587

588

589

590

591

592

593

594

595

596

597

Perfeito L, Fernandes L, Mota C, Gordo I 2007. Adaptive Mutations in Bacteria: High Rate and Small Effects. Science 317:813-815. DOI: 10.1126/science.1142284.

Perfeito L, Sousa A, Bataillon T, Gordo I 2014. Rates of fitness decline and rebound suggest pervasive epistasis. Evolution 68:150-162. DOI: 10.1111/evo.12234.

Sellis D, Callahan BJ, Petrov DA, Messer PW 2011. Heterozygote advantage as a natural consequence of adaptation in diploids. Proceedings of the National Academy of Sciences of the United States of America 108:20666-20671. DOI: 10.1073/pnas.1114573108.

Sniegowski PD, Gerrish PJ, Lenski RE 1997. Evolution of high mutation rates in experimental populations of E. coli. Nature 387:703-705. DOI: 10.1038/42701.

Sousa A, Magalhaes S, Gordo I 2012. Cost of Antibiotic Resistance and the Geometry of Adaptation. Molecular Biology and Evolution 29:1417-1428. DOI: 10.1093/molbev/msr302.

Taddei F, Radman M, Maynard-Smith J, Toupance B, Gouyon PH, Godelle B 1997. Role of mutator alleles in adaptive evolution. Nature 387:700-702. DOI: 10.1038/42696.

Tanaka MM, Bergstrom CT, Levin BR 2003. The evolution of mutator genes in bacterial populations: the roles of environmental change and timing. Genetics 164:843-854.

Tenaillon O 2014. The utility of Fisher's geometric model in evolutionary genetics. Annual Review of Ecology 45:179-201. DOI: 10.1146/annurev-ecolsys-120213-091846.

Tenaillon O, Taddei F, Radmian M, Matic I 2001. Second-order selection in bacterial evolution: selection acting on mutation and recombination rates in the course of adaptation. Research in Microbiologoy 152:11-16.

Torres-Barceló C, Cabot G, Oliver A, Buckling A, MacLean RC 2013. A trade-off between oxidative stress resistance and DNA repair plays a role in the evolution of elevated mutation rates in bacteria. Proceedings of the Royal Society B: Biological Sciences 280:20130007. DOI: $10.1098 / \mathrm{rspb} .2013 .0007$.

Wagner GP, Zhang J 2011. The pleiotropic structure of the genotype-phenotype map: the evolvability of complex organisms. Nature Publishing Group 12:204-213. DOI: $10.1038 / \mathrm{nrg} 2949$.

Wang Z, Liao B-Y, Zhang J 2010. Genomic patterns of pleiotropy and the evolution of complexity. Proceedings of the National Academy of Sciences of the United States of America 107:18034-18039. DOI: 10.1073/pnas.1004666107.

Weinreich DM, Knies JL 2013. Fisher's geometric model of adaptation meets the functional synthesis: data on pairwise epistasis for fitness yields insights into the shape and size of phenotype space. Evolution 67:2957-2972. DOI: 10.1111/evo.12156.

Welch JJ, Waxman D 2003. Modularity and the cost of complexity. Evolution 57:1723-1734.

Wielgoss S, Barrick JE, Tenaillon O, Wiser MJ, Dittmar WJ, Cruveiller S, Chane-Woon-Ming B, Médigue C, Lenski RE, Schneider D 2013. Mutation rate dynamics in a bacterial population reflect tension between adaptation and genetic load. Proceedings of the National Academy of Sciences 110:222-227. DOI: 10.1073/pnas.1219574110.

Zanini F, Brodin J, Thebo L, Lanz C, Bratt G, Albert J 2016. Population genomics of intrapatient HIV-1 evolution. eLife. DOI: 10.7554/eLife.11282.001.

Zeyl C, DeVisser JA 2001. Estimates of the rate and distribution of fitness effects of spontaneous mutation in Saccharomyces cerevisiae. Genetics 157:53-61. 


\section{Figure $\mathbf{1}$ (on next page)}

Dynamics of mean fitness increase under FGM with partial pleiotropy

A) Dynamics of mean fitness of asexual populations with varying degrees of pleiotropy $(m)$. All populations have high complexity $(n=96)$ and distributions of fitness effects (DFEs) with different means are studied. Other parameters are population size $N=10^{4}$, mutation rate $U=0.001$, initial fitness $w_{0}=0.5$. The variance for the mutation effects $\sigma^{2}$ varies as $m$ varies, so that $E(S)$ (which is $-m \sigma^{2}$ in this model) has the value indicated in each panel. $B$ ) The effect of increasing phenotypic complexity $(n)$ on the dynamics of fitness increase. Other population parameters are: $N=10^{4}, U=0.001$, variance $\sigma^{2}=0.004, m=3$ and the initial fitness $w_{0}=0.5$. Short-term dynamics are highlighted as an inset. 
A
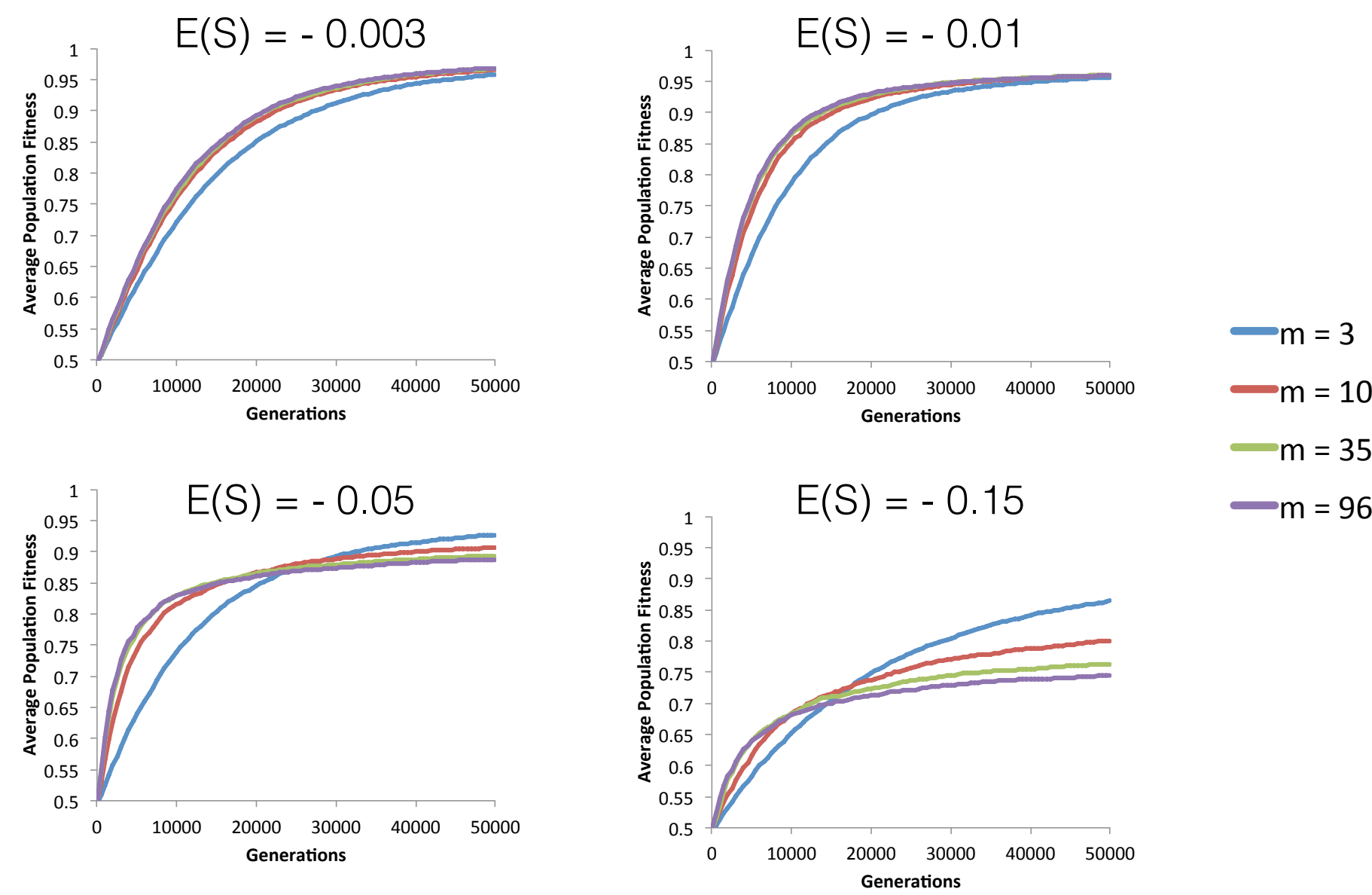

B
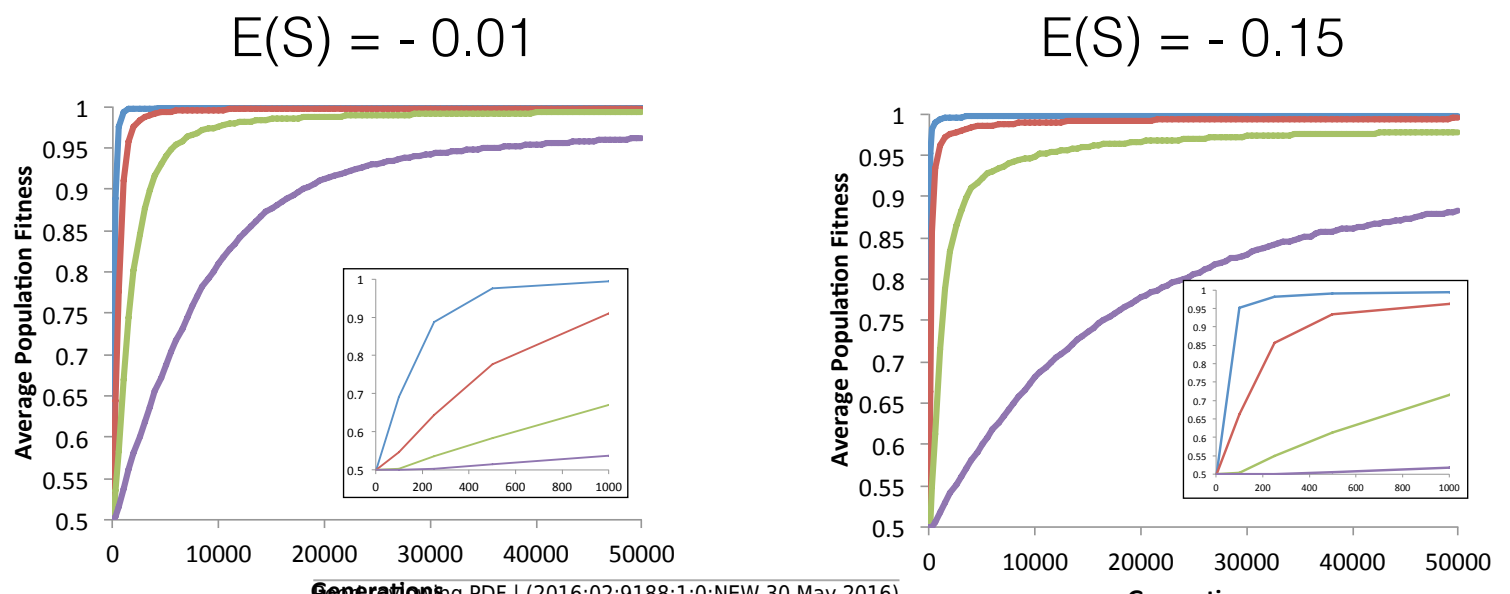

$$
\begin{aligned}
-\mathrm{n} & =3 \\
\mathrm{n} & =10 \\
\mathrm{n} & =30 \\
\mathrm{n} & =90
\end{aligned}
$$




\section{Figure 2 (on next page)}

FGM can lead to simultaneous fixation of mutation cohorts

The probability distribution of the number of mutations fixed during the first fixation event in the adaptive walk, i.e. mutation cohort size. Parameter values used in these simulations are as follows: population size $N=10^{4}$ and initial fitness $w_{0}=0.5$ are the same across all panels; the other parameters vary as indicated in each panel (mutation rate $U$ increases bottom to top from 0.0001 to 0.01 , implying higher levels of clonal interference; phenotypic complexity $n$ (and pleiotropy $m=n$ ) increase from left to right; and, within each panel, $s^{2}$ varies, implying different distributions of arising mutations with mean selective effects of $E(S)$ indicated by the different shades). Data is shown for 100 simulations per combination of parameters. 
Figure 2
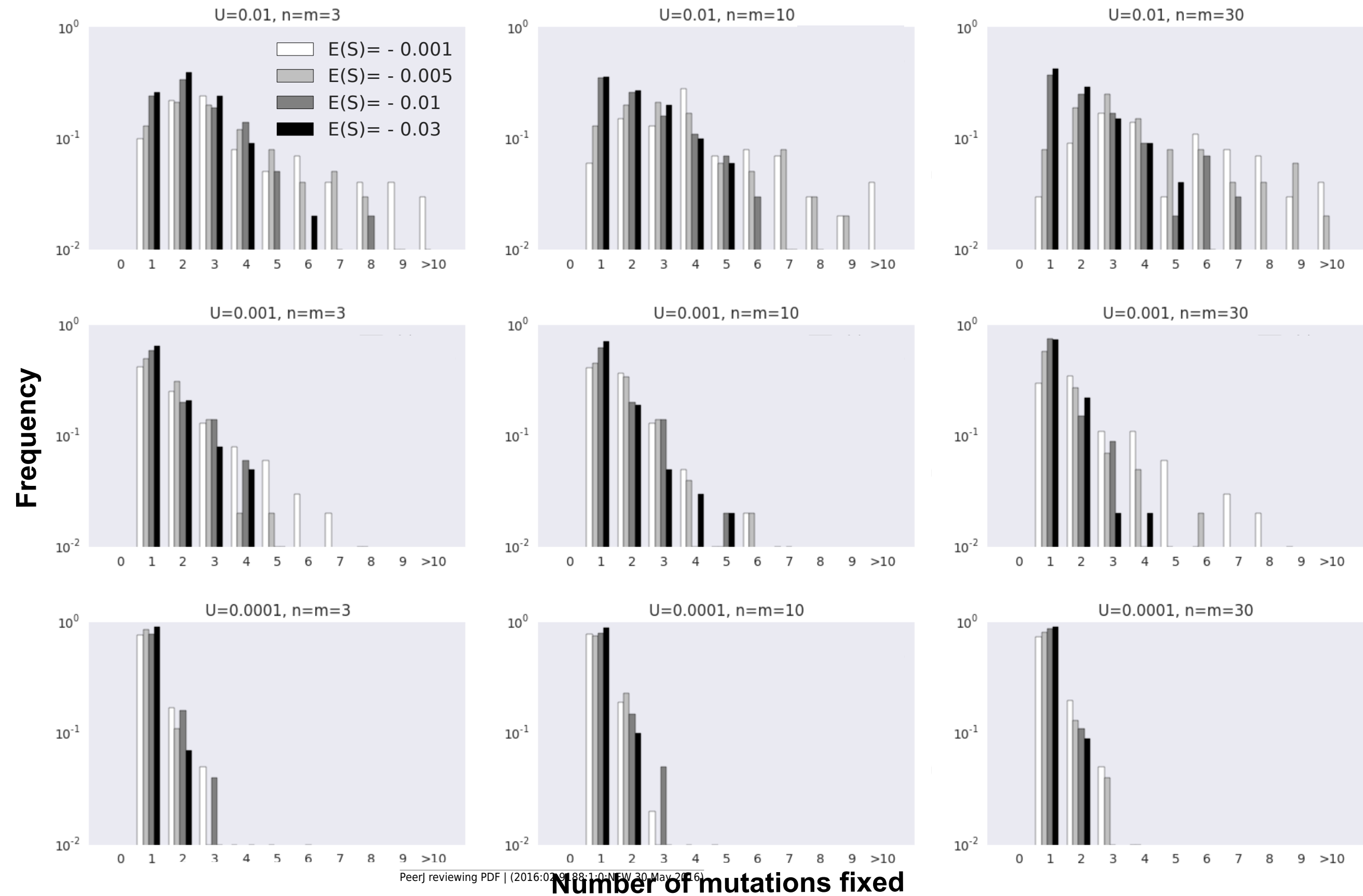
Figure 3 (on next page)

Number of mutations fixed (mutation cohort size) along the adaptive walk

Parameter values are population size $N=10^{4}$, the initial fitness $w_{0}=0.5$, mutation rate $U=0.001$ and other parameters as indicated in each panel (with phenotypic complexity $n$ and mean effect of mutations $E(S)$ taking the values indicated on the left and above panels, respectively). In all panels full pleiotropy is assumed $(m=n)$. Data is shown for 100 simulations per combination of parameters. 


\section{Figure 3}
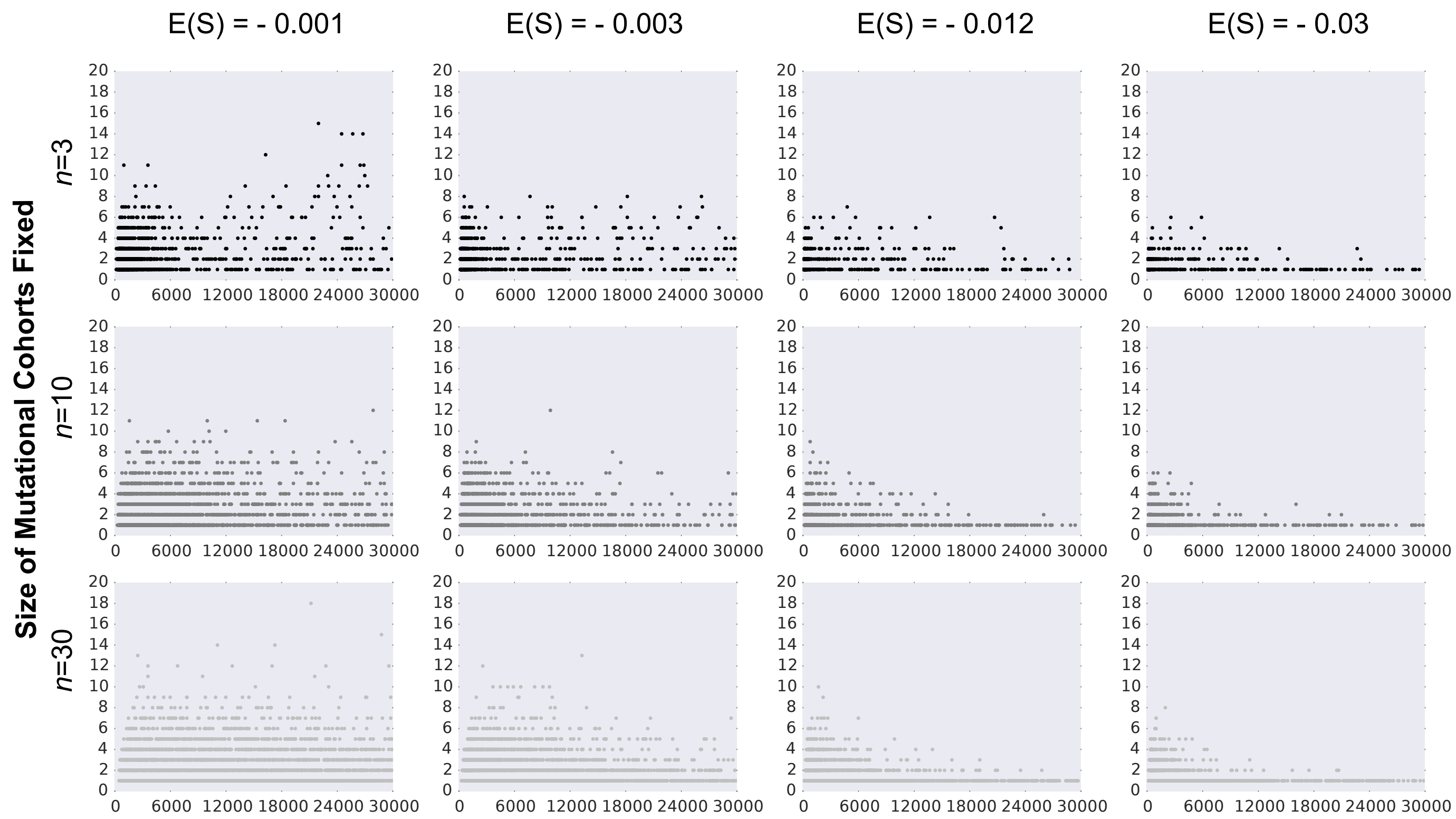

\section{Generations}


Figure 4 (on next page)

Dynamics of frequency change of individual mutations along time across independently evolving populations.

Aggregation of multiple mutations in cohorts can be easily detected by the simultaneous increase in frequency of different mutations (in different colors). Competition between cohorts can be commonly observed during the first 1000 generations of adaptation. Parameter values used are population size $N=10^{5}$, initial fitness $w_{0}=0.5$, mutation rate $U=0.003, n=10, m=3$ and $E(S)=-0.012$. Gaussian noise (mean 0 , variance 0.02 ), mimicking experimental error, was added to the dynamics for increasing visibility and comparison with experimental data. 

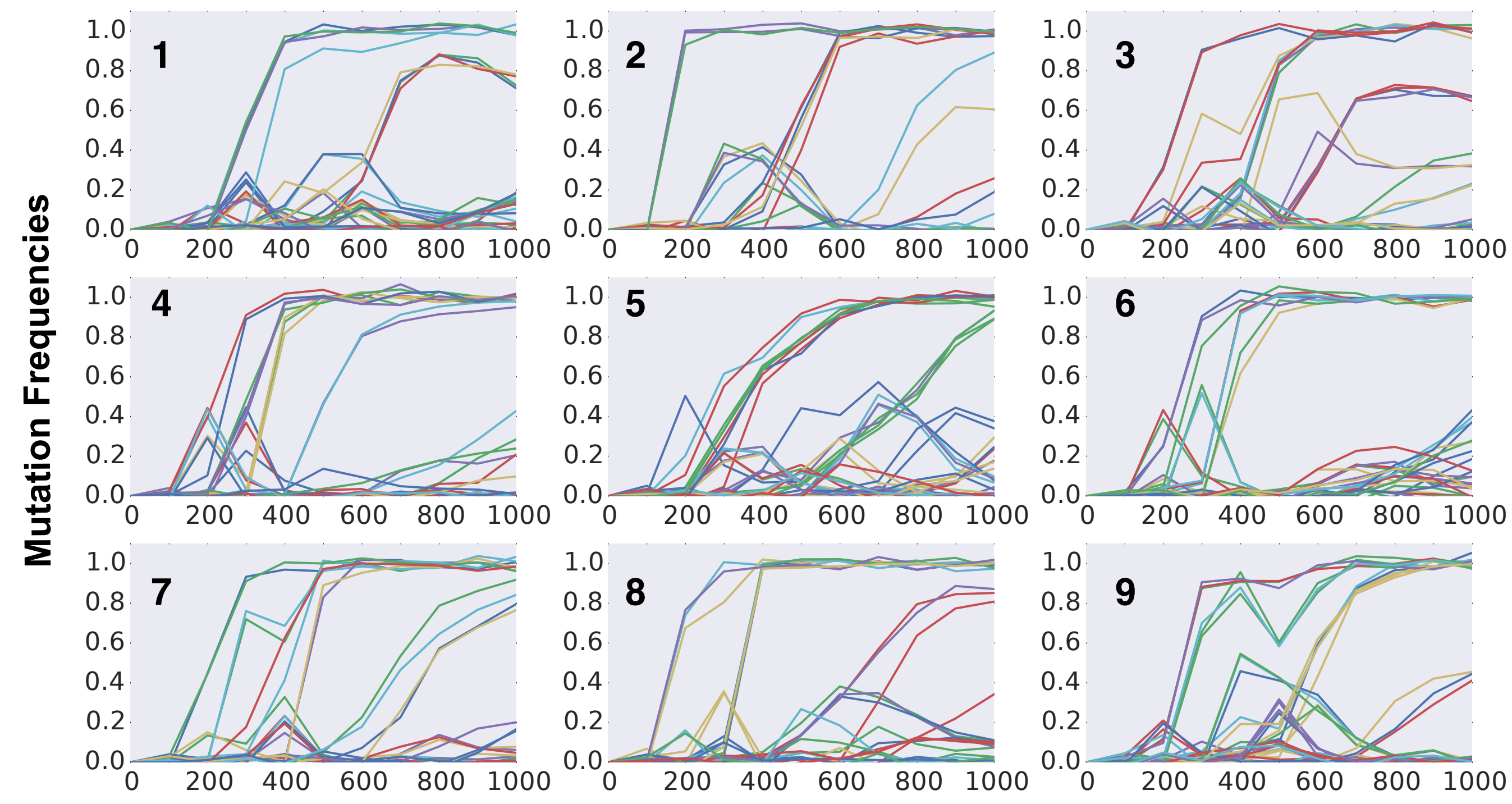

\section{Generations}

